\title{
A 4-COLOR THEOREM FOR SURFACES OF GENUS $g$
}

\author{
KENNETH A. BERMAN AND JEROME L. PAUL
}

(Communicated by Thomas Brylawski)

\begin{abstract}
For $\mathscr{G}$ a set of graphs, define the bounded chromatic number $\chi_{B}(\mathscr{G})$ (resp. the bounded path chromatic number $\chi_{p}(\mathscr{G})$ ) to be the minimum number of colors $c$ for which there exists a constant $M$ such that every graph $G \in \mathscr{G}$ can be vertex $c$-colored so that all but $M$ vertices of $G$ are properly colored (resp. the length of the longest monochromatic path in $G$ is at most $M$ ). For $\mathscr{G}$ the set of toroidal graphs, Albertson and Stromquist [1] conjectured that the bounded chromatic number is 4. For any fixed $g \geq 0$, let $\mathscr{S}_{\boldsymbol{g}}$ denote the set of graphs of genus $g$. The Albertson-Stromquist conjecture can be extended to the conjecture that $\chi_{B}\left(\mathscr{S}_{g}\right)=4$ for all $g \geq 0$. In this paper we show that $4 \leq \chi_{B}\left(\mathscr{S}_{g}\right) \leq 6$. We also show that the bounded path chromatic number $\chi_{p}\left(\overline{\mathscr{S}}_{g}\right)$ equals 4 for all $g \geq 0$. Let $\mu_{c}(g, n)\left(\pi_{c}(g, n)\right)$ denote the minimum $l$ such that every graph of genus $g$ on $n$ vertices can be $c$-colored without forcing $l+1$ monochromatic edges (a monochromatic path of length $l+1)$. We also obtain bounds for $\mu_{c}(g, n)$ and $\pi_{c}(g, n)$.
\end{abstract}

\section{INTRODUCTION AND STATEMENT OF MAIN THEOREMS}

Let $G$ be a connected graph with vertex set $V$ and edge set $E$. By a vertex $c$ coloring of $G$ we will mean any partition of $V$ into $c$ classes $V_{1}, V_{2}, \ldots, V_{c}$. A monochromatic edge is an edge of whose end vertices are colored the same. A monochromatic path is a path all of whose edges are monochromatic. Throughout this paper, a path shall mean a simple path, i.e., no repeated vertices. A vertex is properly colored if it is not incident with any monochromatic edge. Albertson and Stromquist [1] conjectured that there exists a constant $M$ such that any toroidal graph (graph embeddable on the torus) can be 4-colored so that all but $M$ of the vertices are properly colored. This conjecture extends naturally to the set $\mathscr{S}_{g}$ of graphs of arbitrary (fixed) genus $g$, and motivates the following definition for any set $\mathscr{G}$ of graphs. The bounded chromatic number $\chi_{B}(\mathscr{G})$ of $\mathscr{G}$ is the minimum number of colors $c$ for which there exists a constant $M$ such that any graph $G \in \mathscr{G}$ can be $c$-colored so that all but $M$ of the vertices are properly colored. The Albertson-Stromquist conjecture extended to graphs of genus $g$ is equivalent to the conjecture that $\chi_{B}\left(\mathscr{S}_{g}\right)=4$

Received by the editors August 22, 1986 and, in revised form, May 13, 1988.

1980 Mathematics Subject Classification (1985 Revision). Primary 05C15.

Key words and phrases. Vertex coloring, genus $g$, chromatic number. 
(the fact that $\chi_{B}\left(\mathscr{S}_{g}\right)>3$ is immediate: consider copies of $K_{4}$ ). In this paper we prove that $4 \leq \chi_{B}\left(\mathscr{S}_{g}\right) \leq 6$.

A weaker 4-color conjecture for graphs of genus $g$ is obtained by replacing the requirement that the number of improperly colored vertices is bounded by the requirement that the length of the longest monochromatic path is bounded. In this paper we resolve this weaker conjecture by showing that four colors suffice to bound the monochromatic path length. We also show that 4 colors are necessary by constructing a planar graph on $n$ vertices such that any 3-coloring forces a monochromatic path of length $\frac{1}{3} \log _{3}((2 n-5) / 9)$. More formally, define the bounded path chromatic number $\chi_{p}(\mathscr{G})$ to the minimum number of colors $c$ for which there exists a constant $M$ such that every graph $G \in \mathscr{G}$ can be $c$-colored without forcing a monochromatic path of length $M$. The 4-color theorem referred to in the title is the result that $\chi_{p}\left(\mathscr{S}_{g}\right)=4$.

In order to state our results precisely, we will need some additional definitions and notation. Given a graph $G$, let $\mu_{c}(G)\left(\pi_{c}(G)\right)$ denote the minimum total number of monochromatic edges (the minimum length of the longest monochromatic path) over all $c$-colorings of $G$. Define

$$
\begin{gathered}
\mu_{c}(g, n)=\max \left\{\mu_{c}(G): G \in \mathscr{S}_{g} \text { and } G \text { has } n \text { vertices }\right\}, \\
\pi_{c}(g, n)=\max \left\{\pi_{c}(G): G \in \mathscr{S}_{g} \text { and } G \text { has } n \text { vertices }\right\} .
\end{gathered}
$$

Let $t(g)$ be defined recursively by

$$
t(g)=2\left\lceil\frac{t(g-1)+2}{3}\right\rceil(t(g-1)+2)+t(g-1), t(0)=0 .
$$

Note that $t(1)=4$ and $t(g) \leq 2^{2^{g}}-2$ for all $g \geq 2$. In this paper we prove the following two main theorems.

Theorem 1.1. The bounded chromatic number $\chi_{B}\left(\mathscr{S}_{g}\right)$ for the set $\mathscr{S}_{g}$ of graphs of genus $g$ satisfies $4 \leq \chi_{B}\left(\mathscr{S}_{g}\right) \leq 6$. Further, if $\mu_{c}(g, n)$ is given by $(1.1)$ then for all sufficiently large $n$,

$$
\begin{aligned}
& \mu_{2}(0, n)=n-2, \\
& \mu_{3}(0, n)=\left\lfloor\frac{n}{2}\right\rfloor-1, \\
& \mu_{6}(g, n)\left\{\begin{array}{l}
=g, g \leq 1 \\
\leq 84(g-1), g \geq 2 .
\end{array}\right.
\end{aligned}
$$

Theorem 1.2. The bounded-path chromatic number for the set $\mathscr{S}_{g}$ of graphs of genus $g$ equals 4 , i.e., $\chi_{p}\left(\mathscr{S}_{g}\right)=4$. Further, if $\pi_{c}(g, n)$ and $t(g)$ are defined by (1.2) and (1.3) then

$$
\begin{aligned}
& n^{1 / 2}-2 \leq \pi_{2}(0, n) \leq(6 \sqrt{2}+6 \sqrt{3})^{2 / 3} n^{2 / 3}, \\
& \frac{1}{3} \log _{3}\left(\frac{2 n-5}{9}\right) \leq \pi_{3}(0, n) \leq(6 \sqrt{2}+6 \sqrt{3}) n^{1 / 2}, \\
& \pi_{4}(g, n) \leq t(g) .
\end{aligned}
$$


With the observation that $\mu_{c}(g, n) \geq \mu_{c}(0, n)$ and $\pi_{c}(g, n) \geq \pi_{c}(0, n)$ for all geni $g$, it is immediate that the statement $4 \leq \chi_{B}\left(\mathscr{S}_{g}\right) \leq 6$ of Theorem 1.1 follows from (1.4), (1.5), and (1.6), and that the statement $\chi_{p}\left(\mathscr{S}_{g}\right)=4$ of Theorem 1.2 follows from (1.7), (1.8), and (1.9). The lower bound of (1.7) is established by the game of Hex [5]. We establish the lower bound of (1.8) in $\S 4$ by constructing a suitable planar graph. The upper bounds of (1.7) and (1.8) are derived in $\S 5$ using an argument based on the Lipton-Tarjan plane separator theorem [6].

\section{Proof of Theorem 1.1}

In this section we prove Theorem 1.1. For $G$ a simple graph of genus $g$ let $\nu=\nu(G)$ and $\varepsilon=\varepsilon(G)$ denote the number of vertices and number of edges of $G$, respectively. We will employ the following well-known inequality (which is easily obtainable from Euler's polyhedra formula [4, p. 121]).

$$
g \geq \varepsilon / 6-\nu / 2+1
$$

We will first prove (1.4). Let $G$ be any planar graph. Since adding edges can only increase $\mu_{c}(G)$ we may assume that $G$ is a planar triangulation. It is immediate that for every 2-coloring of vertices of $G$, every face contains at least one monochromatic edge. Further, it follows from the 4-color theorem that $G$ can be 2-colored so every face contains at most one monochromatic edge. Now by Euler's polyhedra formula the number of faces of $G$ equals $2 \nu-4$. Thus $\mu_{2}(G)=\nu-2$. This proves (1.4).

We will now prove (1.5). It is an easy consequence of the 4-color theorem that every planar triangulation can be vertex 3-colored so the number of monochromatic edges is at most $\varepsilon / 6$. But by $(2.1) \varepsilon / 6 \leq \nu / 2-1$. It follows that $\mu_{3}(G) \leq \nu / 2-1$, and thus $\mu_{3}(0, n) \leq n / 2-1$.

We will now prove that $\mu_{3}(0, n) \geq\lfloor n / 2\rfloor-1$, for $n \geq 14$. We may assume that $n$ is even. Consider any planar, Eulerian triangulation $T$, (i.e., a planar triangulation such that every vertex has even degree), where $\nu(T)=n / 2+1$. It is easily seen that the faces $F$ of $T$ can be bipartitioned into two classes $F_{1}$ and $F_{2}$ such that no two faces of $F_{1}$ (of $F_{2}$ ) have an edge in common and both $F_{1}$ and $F_{2}$ have cardinality $\nu(T)-2$. Now consider the triangulation $T^{\prime}$ obtained from $T$ by placing a vertex in the interior of every face from $F_{1}$ and joining that vertex with three edges to the three vertice incident with the face. Then $T^{\prime}$ contains $\left|F_{1}\right|=\nu(T)-2$ edge disjoint copies of $K_{4}$ (the complete graph on 4 vertices). Since each copy of $K_{4}$ contains a monochromatic edge no matter how the vertices are 3-colored we have that $\mu_{3}\left(T^{\prime}\right) \geq \nu(T)-2=\nu\left(T^{\prime}\right) / 2-1$, and thus $\mu_{3}(0, n) \geq n / 2-1$.

We complete the proof of Theorem 1.1 by proving inequality (1.6). We will employ the following lemma.

Lemma 2.1. Let $G$ be a graph of genus $g$ such that every vertex of $G$ has degree at least 6 . Then the sum $\sigma_{6}^{\prime}$ of the degrees over all the vertices having degree 
strictly greater than 6 satisfies

$$
\sigma_{6}^{\prime} \leq 84(g-1)
$$

Proof. Let $\nu=\nu(G)$ and $\varepsilon=\varepsilon(G)$ denote the number of vertices and edges of $G$, respectively, and let $\nu_{6}^{\prime}$ denote the number of vertices of degree strictly larger than 6. It is immediate from the definition of $\sigma_{6}^{\prime}$ and $\nu_{6}^{\prime}$ that

$$
\begin{aligned}
& 2 \varepsilon=6\left(\nu-\nu_{6}^{\prime}\right)+\sigma_{6}^{\prime}, \\
& \sigma_{6}^{\prime} \geq 7 \nu_{6}^{\prime} .
\end{aligned}
$$

Combining (2.1), (2.3), and (2.4) yields (2.2) of Lemma 2.1.

We will now prove (1.6) of Theorem 1.1. Let $G$ be any graph of genus $g$. By a sequence of edge contractions each involving an edge incident to a vertex of degree at most 5, we can reduce $G$ to a graph $G$ where $G$ is either (i) a single vertex, or (ii) a graph such that every vertex has degree at least 6. It is immediate that in the former case $\mu_{6}(G)=0$ and in the latter case $\mu_{6}(G)=\mu_{6}\left(G^{\prime}\right)$. We may assume without loss of generality that $G^{\prime}$ is a simple graph since replacing a multi-edge with a single edge does not effect $\mu_{6}\left(G^{\prime}\right)$. Let $G^{\prime \prime}$ be the graph obtained from $G$ by deleting all the vertices of degree strictly greater than 6 and their incident edges. It follows from Lemma 2.1 that $\varepsilon\left(G^{\prime}\right) \leq \varepsilon\left(G^{\prime \prime}\right)+84(g-1)$. Thus, $\mu_{6}\left(G^{\prime}\right) \leq \mu_{6}\left(G^{\prime \prime}\right)+84(g-1)$. Now every vertex of $G^{\prime \prime}$ has degree at most 6 . Therefore, by Brooks' Theorem (see [4, p. 275]) either $G^{\prime \prime}$ can be properly 6-colored, i.e., $\mu_{6}\left(G^{\prime \prime}\right)=0$, and $\mu_{6}\left(G^{\prime}\right) \leq 84(g-1)$, or $G^{\prime \prime}$ is $K_{7}$ the complete graph on 7 vertices. In the latter case, $G$ must also be $K_{7}$ and thus $\mu_{6}\left(G^{\prime}\right)=1$. We now have inequality (1.6) of Theorem 1.1.

\section{UPPER BOUND FOR $\chi_{p}\left(\mathscr{S}_{g}\right)$}

In this section we prove inequality (1.9) of Theorem 1.2 using induction on $g$, from which follows the result that $\chi_{p}\left(\mathscr{S}_{g}\right) \leq 4$. We will do this with the aid of three lemmas.

By the 4-color Theorem (see [2, 3]), $\pi_{4}(0, n)=0$. For $g \geq 1$, assume inductively that (1.9) is true for all geni less than $g$. Consider any graph $G$ of genus $g$. Embed $G$ on an orientable surface $S_{g}$ of genus $g$. A circuit is a connected graph such that each of its vertices has degree two. A circuit $C$ of $G$ is called null homologous if the removal of $C$ from $S_{g}$ disconnects $S_{g}$.

Lemma 3.1. $G$ contains at least one circuit which is not null homologous. Further, if $C$ is any circuit of $G$ which is not null homologous, then the genus of $G-V(C)$, i.e., the graph obtained from $G$ by deleting all the vertices of $C$ and their incident edges, has genus strictly less than that of $G$.

Lemma 3.2. Let $P_{1}, P_{2}, P_{3}$ be three edge disjoint paths in $G$ joining the same two vertices $x$ and $y$. Let $C_{1}, C_{2}, C_{3}$ be the circuits obtained by taking the three 
pairwise unions of these paths. If any two of these circuits is null homologous, then the third is also null homologous.

The proofs of these lemmas are topological in nature and will not be given here. For example Lemma 3.1 follows from the theorem (see $[4, p .95]$ ) that every embedding of a graph $G$ on the surface whose genus is equal to that of $G$, is a 2 -cell embedding.

Lemma 3.3. Let $C$ be a circuit. Then for any positive integer $l$ there exists a 4-coloring of the vertices of $C$ such that for any two distinct vertices $x$ and $y$ of the same color, either $x$ and $y$ lie in a monochromatic path $P$ of length at most $2 l-1$ or both paths joining $x$ and $y$ in $C$ have length at least $3 l+1$.

Lemma 3.3 is easily proved and the proof is left to the reader.

We are now ready to complete the induction step in our proof of inequality (1.9) of Theorem 1.2. Let $C$ be a circuit of $G$ of minimum length which is not null homologous. Set $l=\left\lceil\left(\pi_{4}(g-1, n)+2\right) / 3\right\rceil$. Then by Lemma 3.3 we can 4-color the vertices of $C$ such that each maximal monochromatic path in $C$ has length at most $2 l-1=2\left\lceil\left(\pi_{4}(g-1, n)+2\right) / 3\right\rceil-1$ and such that for any two vertices $x$ and $y$ of the same color which belong to different maximal monochromatic paths in $C$, the two paths in $C$ joining $x$ and $y$ both have length at least $3\left\lceil\left(\pi_{4}(g-1, n)+2\right) / 3\right\rceil+1 \geq \pi_{4}(g-1, n)+3$.

By induction assumption we can 4-color the vertices of $G-V(C)$ such that the longest monochromatic path involving only these vertices has length at most $\pi_{4}(g-1, n)$. This can be done, since by Lemma 3.1, $G-V(C)$ has genus strictly less than $g$.

Now consider a monochromatic path $P$ in $G$ of a maximum length. We will show that $P$ cannot contain two vertices $x$ and $y$ from the circuit $C$ which belong to different maximal monochromatic paths in $C$.

Suppose the contrary is true. Let $P^{\prime}$ denote the portion of the path $P$ joining $x$ and $y$, where $x$ and $y$ are chosen so that the interior vertices of $P^{\prime}$ lie entirely in $G-V(C)$. Then $P^{\prime}$ has length at most $\pi_{4}(g-1, n)+2$. Let $Q_{1}$ and $Q_{2}$ denote the two paths in $C$ joining vertices $x$ and $y$. Both $Q_{1}$ and $Q_{2}$ have length at least $\pi_{4}(g-1, n)+3$. Therefore, the circuit $P^{\prime} Q_{1}$ and the circuit $P^{\prime} Q_{2}$ are both smaller than the circuit $C=Q_{1} Q_{2}$. But circuit $C$ is not null homologous. Therefore, by Lemma 3.2 either $P^{\prime} Q_{1}$ or $P^{\prime} Q_{2}$ is not null homologous. But this contradicts the minimality of $C$. Therefore, $P$ does not contain two vertices from different maximal monochromatic paths in $C$. This implies that $P$ contains at most $2\left\lceil\left(\pi_{4}(g-1, n)+2\right) / 3\right\rceil$ vertices from the circuit $C$. Since $P$ contains at most $\pi_{4}(g-1, n)+1$ consecutive vertices from $G-V(C)$ it follows that $P$ has length at most

$$
2\left\lceil\left(\pi_{4}(g-1, n)+2\right) / 3\right\rceil\left(\pi_{4}(g-1, n)+2\right)+\pi_{4}(g-1, n) .
$$


This completes the proof of inequality (1.9) of Theorem 1.2.

\section{LOWER BOUNDS FOR $\pi_{2}(0, n)$ AND $\pi_{3}(0, n)$}

In this section we will verify the lower bounds for $\pi_{2}(0, n)$ and $\pi_{3}(0, n)$ given in (1.7) and (1.8) of Theorem 1.2. First let us show that $\pi_{2}(0, n)>$ $\sqrt{n}-2$. To do this we consider the planar graph $H$ whose vertex set is the set of all ordered pairs $(x, y)$, where $x, y \in\{0,1,2, \ldots, m-1\}$ and whose edge set is obtained by joining $(x, y)$ to the three vertices $(x+1, y),(x, y+1)$, $(x+1, y+1)$ for $x, y \in\{0,1,2, \ldots, m-2\}$. Consider any 2-coloring of the vertices of $H$ with colors $A$ and $B$. A theorem in [5] states that either there exists a monochromatic path in $H$ colored $A$ joining vertices $\left(0, y_{0}\right)$ and $(m-$ $1, y_{1}$ ) for some $y_{0}, y_{1} \in\{0,1, \ldots, m-1\}$ or there exists a monochromatic path colored $B$ joining vertices $\left(x_{0}, 0\right)$ and $\left(x_{1}, m-1\right)$ for some $x_{0}, x_{1} \in$ $\{0,1, \ldots, m-1\}$. In either case there exists a monochromatic path of length $m-1$. Hence, $\pi_{2}\left(0, m^{2}\right) \geq m-1$. Now consider any positive integer $n$. Then $m^{2} \leq n<(m+1)^{2}$ for some $m$. Thus $\pi_{2}(0, n) \geq \pi_{2}\left(0, m^{2}\right) \geq m-1>\sqrt{n}-2$. This proves the lower bound for $\pi_{2}(0, n)$ given in (1.7).

We will now verify the lower bound for $\pi_{3}(0, n)$ given in (1.8). To do this we consider the sequence of planar triangulations $T_{0}, T_{1}, \ldots$ defined as follows. Triangulation $T_{0}$ is the complete graph $K_{4}$ on 4 vertices. Triangulation $T_{i+1}$ is obtained from $T_{i}$ by placing a new vertex $v$ in every (triangle) face $f$ of $T_{i}$ except the outside face and joining $v$ to the three vertices of $f$ forming three new triangle faces.

For $P$ a path, let $\nu(P)$ denote the number of vertices of $P$. For convenience we will allow a path to have a single vertex or no vertices. We will refer to the path with no vertices as the null path.

Lemma 4.1. Let $C$ be any 3-coloring of the triangulation $T_{i}$ with the colors $1,2,3$. Then there exists a face $f_{i}$ of $T_{i}$ and three monochromatic paths $P_{1}^{(i)}, P_{2}^{(i)}, P_{3}^{(i)}$ colored $1,2,3$, respectively, such that each of these paths has an end vertex incident with face $f_{i}$ and such that

$$
\nu\left(P_{1}^{(i)}\right)+\nu\left(P_{2}^{(i)}\right)+\nu\left(P_{3}^{(i)}\right) \geq i+3 .
$$

(Note that path $P_{j}$ will be the null path if no vertex of face $f_{i}$ is colored $j, j=$ $1,2,3$.)

Proof. We prove the lemma by induction. It is easily verified for triangulation $T_{1}\left(=K_{4}\right)$. Assume the lemma is true for the triangulation $T_{i}$. We will show that this implies it is true for the triangulation $T_{i+1}$. Consider any 3-coloring $C$ of $T_{i+1}$ with the colors 1,2,3. This induces (in the obvious way) a 3-coloring, say $C^{\prime}$, of $T_{i}$. By the induction assumption there exists a face $f_{i}$ of $T_{i}$ and three monochromatic paths $P_{1}^{(i)}, P_{2}^{(i)}, P_{3}^{(i)}$ of colors $1,2,3$, respectively, such that each of these paths has an end vertex incident with face $f_{i}$ and such that inequality (4.1) holds. Let a,b,c denote the three vertices of face $f_{i}$. 
In obtaining $T_{i+1}$ from $T_{i}$ a vertex, say $w$, is placed in face $f_{i}$ and this vertex is joined with three edges to the three vertices a,b,c forming three new triangle faces. Let $f_{a}$ be the face not incident with $a, f_{b}$ the face not incident with $b$, and $f_{c}$ the face not incident with $c$. There are two cases to consider. First suppose that vertex $w$ is colored differently from each of the vertices a,b,c. Assume without loss of generality that the color of $w$ is 1 . This implies that $P_{1}^{(i)}$ is the null path (since none of the vertices of $f_{i}$ are colored 1). Let $P_{1}^{(i+1)}$ be the path consisting of the single vertex $w$ and let $P_{2}^{(i+1)}=P_{2}^{(i)}, P_{3}^{(i+1)}=P_{3}^{(i)}$. Since $\nu\left(P_{1}^{(i+1)}\right)=1$ and $\nu\left(P_{1}^{(i)}\right)=0$ it follows from inequality (4.1) that

$$
\nu\left(P_{1}^{(i+1)}\right)+\nu\left(P_{2}^{(i+1)}\right)+\nu\left(P_{3}^{(i+1)}\right) \geq i+4 .
$$

One of the faces $\left\{f_{a}, f_{b}, f_{c}\right\}$ contains the vertex $w$ and an end vertex from each of the paths $P_{2}^{(i)}$ and $P_{3}^{(i)}$. Let $f_{i+1}$ denote this face. Then all three paths $P_{1}^{(i+1)}, P_{2}^{(i+1)}, P_{3}^{(i+1)}$ have an end vertex incident with face $f_{i+1}$ so that the conclusions of Lemma 4.1 are satisfied.

We now consider the second case where vertex $w$ is colored the same as one of the vertices a,b,c. Assume without loss of generality that $a$ and $w$ are colored the same and that the color is 1 . Further we may assume that $a$ is an end vertex of the path $P_{1}^{(i)}$. Let $P_{1}^{(i+1)}$ be the path consisting of path $P_{1}^{(i)}$ together with the edge $a w$ and the vertex $w$, i.e.,

$$
P_{1}^{(i+1)}=P_{1}^{(i)} w .
$$

Let $P_{2}^{(i+1)}=P_{2}^{(i)}$ and $P_{3}^{(i+1)}=P_{3}^{(i)}$. Since $\nu\left(P_{1}^{(i+1)}\right)=\nu\left(P_{1}^{(i)}\right)+1$ it follows from inequality (4.1) that

$$
\nu\left(P_{1}^{(i+1)}\right)+\nu\left(P_{2}^{(i+1)}\right)+\nu\left(P_{3}^{(i+1)}\right) \geq i+4 .
$$

Let $f_{i+1}$ denote the face from $\left\{f_{a}, f_{b}, f_{c}\right\}$ which does not contains the edge $a w$. It is clear that paths $P_{1}^{(i+1)}, P_{2}^{(i+1)}, P_{3}^{(i+1)}$ each have an end vertex incident with face $f_{i+1}$, so that the conclusions of Lemma 4.1 are satisfied in this case also. This completes the inductive proof of Lemma 4.1. The following corollary follows immediately.

Corollary 4.2. For any 3-coloring of $T_{i}$ there exists a monochromatic path of length at least $i / 3$.

We are now ready to prove the bound for $\pi_{3}(0, n)$ given in (1.8). It is easily verified that the number of vertices of $T_{i}$ is $\left(3^{i+1}+5\right) / 2$. Thus Corollary 4.2 yields $\pi_{3}\left(0,\left(3^{i+1}+5\right) / 2\right) \geq i / 3$. Now let $n$ be any positive integer $n \geq 4$. Choose $i$ such that $\left(3^{i+1}+5\right) / 2 \leq n<\left(3^{i+2}+5\right) / 2$. Then

$$
\pi_{3}(0, n) \geq \pi_{3}\left(0, \frac{3^{i+1}+5}{2}\right) \geq \frac{i}{3} \geq \frac{1}{3} \log _{3} \frac{(2 n-5)}{9} \text {. }
$$


5. UPPER BOUNDS FOR $\pi_{2}(0, n)$ AND $\pi_{3}(0, n)$

In this section we verify the upper bounds for $\pi_{2}(0, n)$ and $\pi_{3}(0, n)$ given by (1.7) and (1.8) in Theorem 1.2. To do so we will employ two lemmas.

For $G$ a graph, not necessarily connected, let $\lambda(G)$ denote the largest number of vertices in a component of $G$. For $U$ a subset of the vertices of $G$, let $G[U]$ denote the subgraph induced by the vertices in $U$.

Lemma 5.1. Let $G$ be any planar graph on $n$ vertices and let $i$ be any positive integer. Then there exists a bipartition (2-coloring) of the vertex set into two parts $V_{1}=V_{1}^{(i)}$, and $V_{2}=V_{2}^{(i)}$ such that

$$
\begin{gathered}
\left|V_{1}\right| \leq 2 \sqrt{2}\left(\frac{\left(\frac{3}{2}\right)^{(i+1) / 2}-1}{\left(\frac{3}{2}\right)^{1 / 2}-1}-\left(\frac{3}{2}\right)^{1 / 2}\right) n^{1 / 2} \leq(6 \sqrt{2}+4 \sqrt{3})\left(\frac{3}{2}\right)^{i / 2} n^{1 / 2}, \\
\lambda\left(G\left[V_{2}\right]\right) \leq\left(\frac{3}{2}\right)^{-i} n .
\end{gathered}
$$

Proof. The Lipton-Tarjan separator theorem for planar graphs (see [6]) states that the vertex set of any planar graph on $n$ vertices can be partitioned into sets $A, B, C$ such that $A$ contains no more than $2 \sqrt{2} \sqrt{n}$ vertices, no edge joins a vertex in $B$ with a vertex in $C$, and neither $B$ nor $C$ contains more than $2 n / 3$ vertices. This immediately yields the case $i=1$ of Lemma 5.1 .

We will show by induction that if Lemma 5.1 is true for $i=j$ then it is true for $i=j+1$. By induction assumption there exists a set $V_{1}^{(j)}$ of vertices of cardinality at most $\left.2 \sqrt{2}\left(\left(\frac{3}{2}\right)^{(j+1) / 2}-1\right)\left(\left(\frac{3}{2}\right)^{1 / 2}-1\right)^{-1}-\left(\frac{3}{2}\right)^{1 / 2}\right) n^{1 / 2}$ such that every component of $G-V_{1}^{(j)}\left(=G\left[V_{2}^{(j)}\right]\right)$ has at most $\left(\frac{2}{3}\right)^{j} n$ vertices. Let $C_{1}, C_{2}, \ldots, C_{\gamma}$ denote the components of $G-V_{1}^{(j)}$ having greater than $\left(\frac{2}{3}\right)^{j+1} n$ vertices and let $n_{1}, n_{2}, \ldots, n_{\gamma}$ denote the number of vertices in $C_{1}, C_{2}, \ldots, C_{\gamma}$, respectively. Then, $n_{1}+n_{2}+\cdots+n_{\gamma} \leq n$ and $\left(\frac{2}{3}\right)^{j+1} n<n_{k} \leq$ $\left(\frac{2}{3}\right)^{j} n$ for $k=1,2, \ldots, \gamma$. These inequalities imply that $\gamma \leq\left(\frac{3}{2}\right)^{j+1}$.

Now by the Lipton-Tarjan separator theorem there exists for each $k \in$ $\{1,2, \ldots, \gamma\}$ a subset $A_{k}$ of the vertices of $C_{k}$ such that $A_{k}$ has cardinality at most $2 \sqrt{2} \sqrt{n}_{k}$ and such that every component of $C_{k}-A_{k}$ has cardinality at most $\left(\frac{2}{3}\right)^{j+1} n$. Set $V_{1}^{(j+1)}=V_{1}^{(j)} \cup A_{1} \cup A_{2} \cdots \cup A_{k}$. Then each of the components of $G-V_{1}^{(j+1)}\left(=G\left[V_{2}^{(j+1)}\right]\right)$ has at most $\left(\frac{2}{3}\right)^{j+1} n$ vertices. Further

$$
\left|V_{1}^{(j+1)}\right| \leq\left|V_{1}^{(j)}\right|+2 \sqrt{2}\left(\sqrt{n}_{1}+\sqrt{n}_{2}+\cdots+\sqrt{n}_{\gamma}\right) .
$$

But

$$
\begin{aligned}
\sqrt{n_{1}}+\sqrt{n_{2}}+\cdots+\sqrt{n_{\gamma}} & \leq \sqrt{\gamma} \sqrt{n_{1}+n_{2}+\cdots+n_{\gamma}} \\
& \leq\left(\frac{3}{2}\right)^{(j+1) / 2} \sqrt{n}
\end{aligned}
$$


Therefore,

This proves Lemma 5.1.

$$
\begin{aligned}
\left|V_{1}^{(j+1)}\right| & \leq 2 \sqrt{2}\left(\frac{\left(\frac{3}{2}\right)^{(j+1) / 2}-1}{\left(\frac{3}{2}\right)^{1 / 2}-1}+\left(\frac{3}{2}\right)^{(j+1) / 2}-\left(\frac{3}{2}\right)^{1 / 2}\right) n^{1 / 2} \\
& =2 \sqrt{2}\left(\frac{\left(\frac{3}{2}\right)^{(j+2) / 2}-1}{\left(\frac{3}{2}\right)^{1 / 2}-1}-\left(\frac{3}{2}\right)^{1 / 2}\right) n^{1 / 2} .
\end{aligned}
$$

Lemma 5.2. Let $G$ be a planar graph on $n$ vertices and let $i, j$ be any two positive integers. Then there exists a tripartition of the vertex set of $G$ into three parts $W_{1}, W_{2}, W_{3}$ such that

$$
\begin{gathered}
\left|W_{1}\right| \leq(6 \sqrt{2}+4 \sqrt{3})^{3 / 2}\left(\frac{3}{2}\right)^{(i / 4+j / 2)} n^{1 / 4}, \\
\lambda\left(G\left[W_{2}\right]\right) \leq(6 \sqrt{2}+4 \sqrt{3})\left(\frac{3}{2}\right)^{(i / 2-j)} n^{1 / 2}, \\
\lambda\left(G\left[W_{3}\right]\right) \leq\left(\frac{3}{2}\right)^{-i} n .
\end{gathered}
$$

Lemma 5.2 is obtained by first applying Lemma 5.1 to the graph $G$ with bipartition $V_{1}=W_{1} \cup W_{2}$ and $V_{2}=W_{3}$ and then applying Lemma 5.1 to the graph $G\left[W_{1} \cup W_{2}\right]$.

We are now ready to prove the upper bounds for $\pi_{2}(0, n)$ and $\pi_{3}(0, n)$ given in (1.7) and (1.8). Lemmas 5.1 and 5.2 imply that for any positive integers $i$ and $j$

$$
\pi_{2}(0, n) \leq \max \left\{(6 \sqrt{2}+4 \sqrt{3})\left(\frac{3}{2}\right)^{i / 2} n^{1 / 2},\left(\frac{3}{2}\right)^{-i} n\right\}
$$

$$
\begin{aligned}
& \pi_{3}(0, n) \\
& \quad \leq \max \left\{(6 \sqrt{2}+4 \sqrt{3})^{3 / 2}\left(\frac{3}{2}\right)^{(i / 4+j / 2)} n^{1 / 4},(6 \sqrt{2}+4 \sqrt{3})\left(\frac{3}{2}\right)^{i / 2-j} n^{1 / 2},\left(\frac{3}{2}\right)^{-i} n\right\} .
\end{aligned}
$$

To minimize the right-hand side of (5.1), choose $i$ such that

$$
(6 \sqrt{2}+4 \sqrt{3})^{2}\left(\frac{3}{2}\right)^{3 i-2} \leq n<(6 \sqrt{2}+4 \sqrt{3})^{2}\left(\frac{3}{2}\right)^{3 i+1} \text {. }
$$

Then substitution and simplification yields

$$
\pi_{2}(0, n) \leq(6 \sqrt{2}+6 \sqrt{3})^{2 / 3} n^{2 / 3} \text {. }
$$

To minimize the right-hand side of (5.2), choose $i$ such that

$$
(6 \sqrt{2}+4 \sqrt{3})^{2}\left(\frac{3}{2}\right)^{2 i-1} \leq n<(6 \sqrt{2}+4 \sqrt{3})^{2}\left(\frac{3}{2}\right)^{2 i+1} \text {, }
$$

and choose $j$ such that

$$
(6 \sqrt{2}+4 \sqrt{3})^{2}\left(\frac{3}{2}\right)^{4 j-3} \leq n<(6 \sqrt{2}+4 \sqrt{3})^{2}\left(\frac{3}{2}\right)^{4 j+1} .
$$

Then, $\pi_{3}(0, n) \leq(6 \sqrt{2}+6 \sqrt{3}) n^{1 / 2}$.

This verifies the upper bounds of (1.7) and (1.8) and completes the proof of Theorem 1.2.

\section{ACKNOWLEDGMENT.}

We would like to thank Noga Alon for helpful discussions. 


\section{REFERENCES}

1. M. Albertson and W. Stromquist, Locally planar toroidal graphs are 5-colorable, Proc. Amer. Math. Soc. 84 (1982), 449-456.

2. K. Appel and W. Haken, The solution of the four-color map problem, Sci. Amer. 237 (1977), 108-121.

3. K. Appel, W. Haken, and J. Koch, Every planar map is four-colorable, Illinois J. Math. 21 (1977), 429-567.

4. M. Behzad, G. Chartrand, and L. Lesniak-Foster, Graphs and Digraphs, Prindle, Weber \& Schmidt, Boston, 1979.

5. D. Gale, The game of Hex and the Brouwer fixed point theorem, Amer. Math. Monthly 86 (1979), 818-827.

6. R. Lipton and R. Tarjan, A separator theorem for planar graphs, SIAM J. Appl. Math. 36 (1979), 177-189.

Department OF Computer Science, University of Cincinnati, Cincinnati, OHIO 45221-0008 